\title{
VANET based Real-Time Intelligent Transportation System
}

\author{
Pallavi A. Targe \\ PG Student, Department of E\&TC \\ K. K. Wagh Institute of Engineering Education, \\ Nasik, Maharashtra, INDIA
}

\author{
M. P. Satone, $\mathrm{PhD}$ \\ Professor, Department of E\&TC \\ K. K. Wagh Institute of Engineering Education, \\ Nasik, Maharashtra, INDIA
}

\begin{abstract}
Due to the rapid growth in the field of Vehicular Ad-hoc Networks (VANET), they are being used for various applications. Intelligent Transportation systems (ITS) are one of the most important applications of VANET which consist of Vehicle-to-vehicle and vehicle-to-infrastructure communications based on wireless local area networks. Traffic accidents and congestion problems are increasing day by day. Transportation sector is significantly stressed, because of vast number of vehicles on road leading to more accidents and fatalities, adverse environmental and economic impact. Intelligent Transportation Systems is one of the solutions to all these problems, which will help to minimize traffic related problems to a great extent and can make our life easier. This paper gives an overview of past few techniques in this field and presents a real-time intelligent transportation system based on VANET. Proposed system is based on RFID and ARM controller to minimize traffic congestion with proper signal control, and then with the help of android mobile application, path planning will take place to provide vehicles with suitable path having minimum traffic to their destination. Proposed system can help to avoid congestion and can find better routes by real time data. This saves both time and fuel and has significant economic advantages.
\end{abstract}

\section{Keywords}

ITS, VANET, RFID, ARM, signal control, android mobile application, path planning.

\section{INTRODUCTION}

As an important societal problem, traffic congestion has received considerable attention. 2007 Urban Mobility survey stated that traffic congestion causes 4.2 billion hours of extra travel every year in U.S. which nearly accounts for 2.9 billion extra gallons of gasoline. Though many existing navigation devices have functionalities to provide an optimal end-to-end path, congestion problems have not been fully resolved [1]. Many conventional techniques still face a lot of technical challenges, as Google Maps involve existing networks for individual path planning to avoid traffic congestion. Although, these provided services are very costly, they are unable to make quick response to an emergency situation caused by any accident or incident. Main reason for this imperfection is lack of real-time traffic information. Thus, to improve the performance of path planning, it is necessary to study how to efficiently collect and utilize real-time traffic information for traffic congestion avoidance and path planning [2], [3].

Intelligent Transportation Systems (ITS) describe technology applied to vehicles as well as infrastructure to transfer information between them for improving safety, productivity and minimizing traffic congestion. This includes applications involving vehicle-to-vehicle as well as vehicle to infrastructure communications such as traffic management systems, cooperative ITS, information and warning systems [3].

Intelligent transportation systems (ITS) are advanced applications for providing innovative services which are related to transport and traffic management and allow users to be better informed and make safer, coordinated, and smarter use of transport networks [1].

Vehicular Ad Hoc Networks (VANETs) are a key component of intelligent transportation systems which are formed by applying the principles of Mobile Ad Hoc Networks (MANETs). VANETs support a wide range of applications from one hop dissemination to multihop dissemination. Most of the concerns of interest in mobile ad hoc networks are of interest in Vehicular ad hoc networks, but their details may differ [1].

VANETs can provide enhanced communication capabilities to collect real-time traffic related information for cost effective and real-time traffic information delivery [5]. Both vehicle-tovehicle (V2V) and vehicle-to-roadside (V2R) communications are supported in VANETs to efficiently collect traffic updates from/to vehicles and roadside units. As a result, collected real-time traffic information can be utilized for freeway traffic flow management, path planning, and vehicle localization [4]. However, most of the works assume that the incorporated techniques have small delivery delay for real-time collection of traffic information. As VANETs rely on short-range communication, the transmission delay cannot be neglected. Therefore, it is necessary to study how the transmission performance can affect the performance of path planning and how to design different transmission mechanisms to reduce delay when it cannot be neglected [6], [7].

We propose a real-time algorithm that utilizes VANET communication capabilities to avoid vehicles from traffic related congestion. Both the network spatial utilization as well as the vehicle travel cost are considered equally important to balance both the drivers' preferences and overall network smoothness.

\section{RELATED WORK}

Traffic congestion can become a major problem in transportation which can result in late arrivals as well as additional cost for drivers. However, we can reduce this cost by navigation or path planning which will help to avoid traffic congestion. For example, vehicles can be rerouted with the shortest path to their destination based on GPS navigation [9], accident duration prediction [13], advance route reservation [10] etc. However, these approaches are not effective to make quick response to an emergency caused by sudden accident 
since update of the traffic information is not real-time. Thus, the real-time traffic information is important.

To collect real time traffic flow, most existing techniques in ITS usually rely on cellular systems and loop detectors. Cell phones with their cellular access have been investigated to collect real-time traffic information. For continuous traffic flow measurement and monitoring, a traffic management system with loop detectors was introduced. However, because of some drawbacks, applications of cellular systems with loop detectors are lacking somewhere. As cellular systems are not dedicated to traffic data collection, these services can be costly, and traffic congestion can become even worse for high volume of traffic flow. The deployment expense for loop detectors can also be very high [12].

VANET can make real-time information delivery much quicker and cheaper with their Vehicle to vehicle and vehicle to infrastructure communications, to become more efficient than existing systems. Moreover, roadside units in VANET are capable of enhancing timeliness of data collection, and hence it is possible to perform effective path planning for a group of vehicles [15].

Many techniques have considered real-time vehicle path planning, a distributed path planning technique is studied to avoid traffic congestion using real-time traffic data, with increased amount of traffic flow. A navigation system worked on saving gasoline for individual vehicle, and also to avoid traffic congestion. However, individual path planning can also introduce additional amount of traffic congestion because of human uncoordinated selfish behaviors. Thus, to balance network traffic, paths of different vehicles should be jointly planned. In multivehicle path planning, drivers' preference or average travel cost is not considered. Furthermore, how communications in VANETs can affect path planning algorithm is still not clear [15]. Many techniques have been developed in the field of Vehicular Ad Hoc Networks. Some of the earlier techniques includes:

Fleetnet concentrated on various research areas and supported by some manufacturers as well as academic centers in Europe. It was based on vehicle to vehicle and vehicle to roadside communication with main objective of driver assistance, safety and security. It includes character description of VANET, suggesting new routing as well as MAC protocols, with exploration of some other technologies in wireless and Ad-Hoc [14].

VII (Vehicle Infrastructure Integration) has a main objective to deploy communications infrastructure that will support both vehicle to infrastructure as well as vehicle to vehicle communications, for a variety of applications. Principal applications are under development such as noticing drivers for unsafe conditions and collision which can be avoided [14].

VSC (Vehicle Safety Communication) has run many experiments with National Highway Traffic and Safety Administration (NHTSA). Its objective is to improve safety with the use of Dedicated Short Range Communication (DSRC), with requirements and parameters for safety applications, traveler information and fuel efficiency [14].

IVI (Intelligent Vehicle Initiative) has a goal to decrease severity of crashes by avoiding driver mistakes. It is based on vehicle to vehicle communication [14].

C2C (Car-to-Car Communication) was started in 2001 and has a main objectives of development and release of an open European standard with driver assistance and safety applications. It is also based on vehicle to vehicle communication [14].

Goal of NOW (Network-on-Wheels) was to perform optimum protocols for transferring messages in addition to methods of securing data transfer. It is also based on vehicle to vehicle communication [14].

CVIS (Cooperative Vehicle Infrastructure System) was developed in 2006 to allow cars to communicate with each other and with the nearby roadside infrastructure with goal of road safety and security. It is based on vehicle to vehicle and vehicle to infrastructure communication [14].

EVITA (E-safety vehicle intrusion protected application) was designed to verify architecture for automotives with security components as well as sensitive data protecting. In addition, it also protects an intra-vehicle communication. It is based on vehicle to vehicle and vehicle to infrastructure communication [14].

Some of the recent techniques includes: Realization of bus transportation system, which uses Radio Frequency Identification (RFID) and wireless sensor technology to enhance public transportation system [17].

Intelligent transportation system with wireless sensor network monitors online travel buses in real-time, to achieve the purpose of intelligent management and traffic measurement [19].

A predictive road traffic management system (PRTMS) is based on Vehicular Ad-hoc Network (VANET). PRTMS uses a novel communication method to estimate future traffic flow at different locations based on a linear prediction algorithm. With the help of this prediction, a central controller will start reducing the congestion level by re-planning the path for vehicles [18].

Another technique has vehicles equipped with onboard unit to enable V2V communication for delivering the vehicle information (for e.g., vehicle location, density, and velocity) [1]. When any vehicle senses accident related congestion, warning message will get generated and shared among all vehicles as well as with nearest roadside unit via V2R communication.

\section{PROPOSED SYSTEM}

Overview of the main system is shown below in fig.1

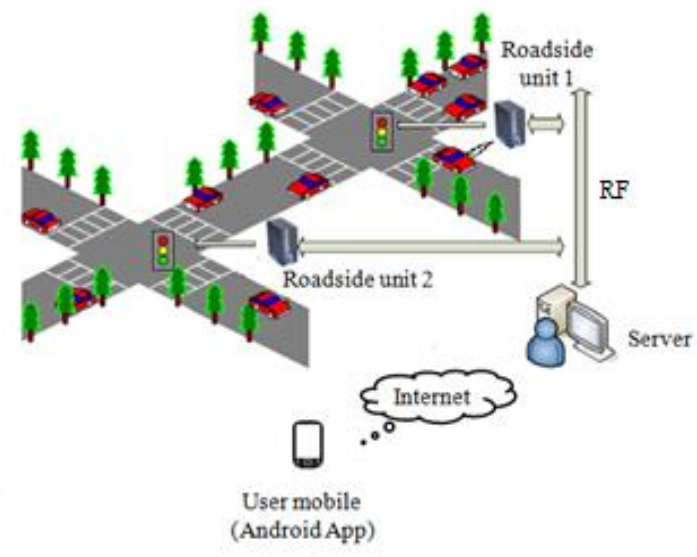

Fig 1: Overview of the system 


\subsection{Signal Monitoring and Control}

Signal monitor and control is becoming more complicated in today's life, as nobody is ready to wait on signal until it turns into green, which leads to a large number of accidents and traffic congestion. This problem can be overcome by controlling the traffic signal on real time using Vehicular AdHoc Network protocol, so nobody has to wait unnecessarily on signal, and it will be helpful to minimize accidents at signal.

\subsection{Path Planning}

Traffic congestion problems have become a very crucial problem, because of rapid growth of car ownership. These cause great inconvenience to people in their daily life and also bring environmental pollution, waste of energy, traffic jams. This automatically affects the improvement of people's living standard, social and economic development. Path planning for urban traffic can solve the problem of road congestion and travel inconvenience.

\subsection{System View}

Block diagram of roadside unit is shown in Fig. 2, every vehicle will be equipped with RFID tag which is read by corresponding roadside unit having RFID reader. By sensing all vehicles present at particular signal by their corresponding RFID tag, roadside unit will collect the information about strength of traffic on that signal node, and this information is processed by controller.

Traffic cycle will be controlled by sensing the amount of traffic at every signal node (i.e. at each junction) and accordingly appropriate time will be allocated to each junction, namely, signal node $\mathrm{A}$, signal node $\mathrm{B}$, signal node $\mathrm{C}$ and signal node D. This particular time slot may vary depending upon the quantity of vehicles at that node, so nobody has to wait unnecessarily on signal, and it will be helpful to minimize accidents.

Traffic information collected by each roadside unit will be provided to the central server via RF communication which will get stored in our database. Block diagram of server unit is shown in Fig. 3, users can have access to this central server via an android mobile application. By providing particular source and destination in this android application, user will get recommended with a path having minimum traffic. This will greatly reduce the problems of road congestion, traffic jams, travel inconvenience etc.

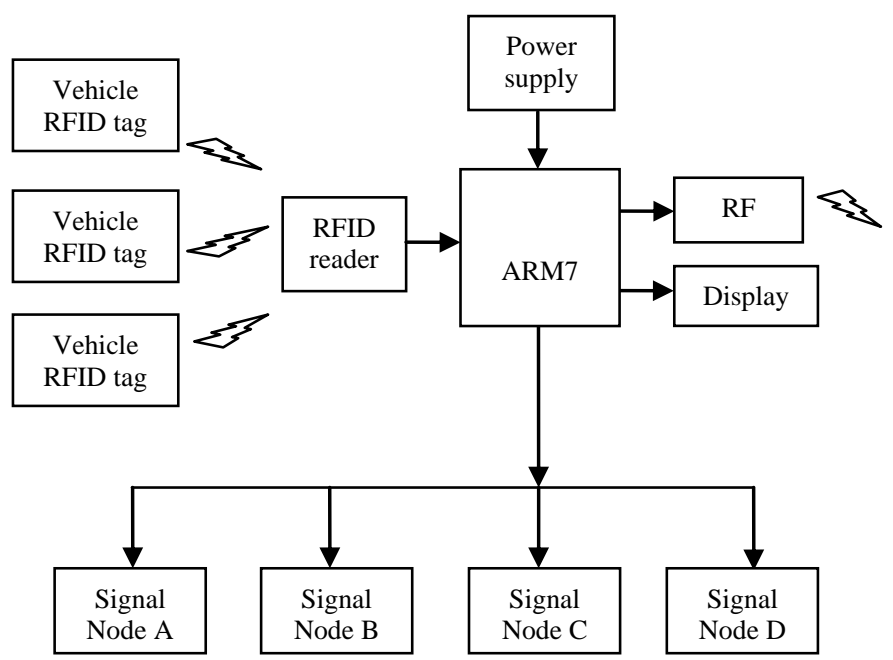

Fig 2: Block diagram of roadside unit

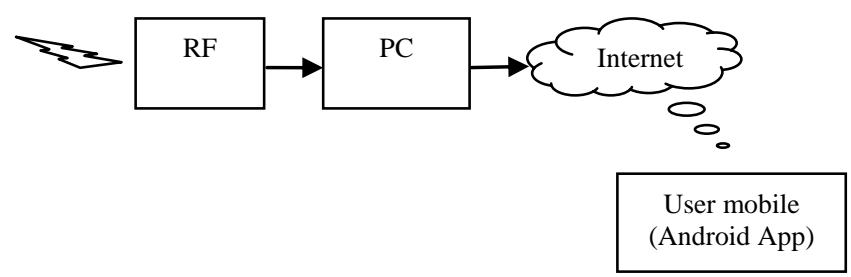

Fig 3: Block diagram of server unit

Proposed system is a privacy preserving system, as it senses vehicles by their RFID tag and processes this information only. It does not capture any other details of these vehicles, hence privacy is maintained.

\section{MOBILE APPLICATION}

The android application is developed to support best path planning strategy. Figure 4(a) will be the first view of our android application.

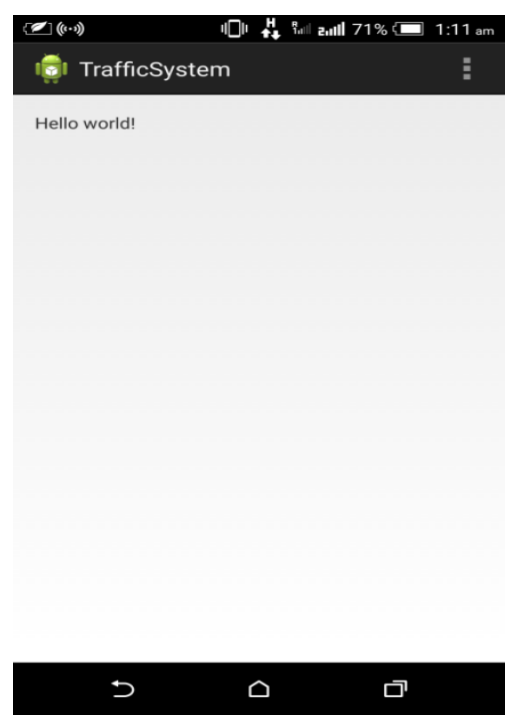

(a)

Then the application will ask for either admin login or user login. As shown in figure 4(b) if we select admin login then we have to provide email address and password of our main server from where we are providing the database.

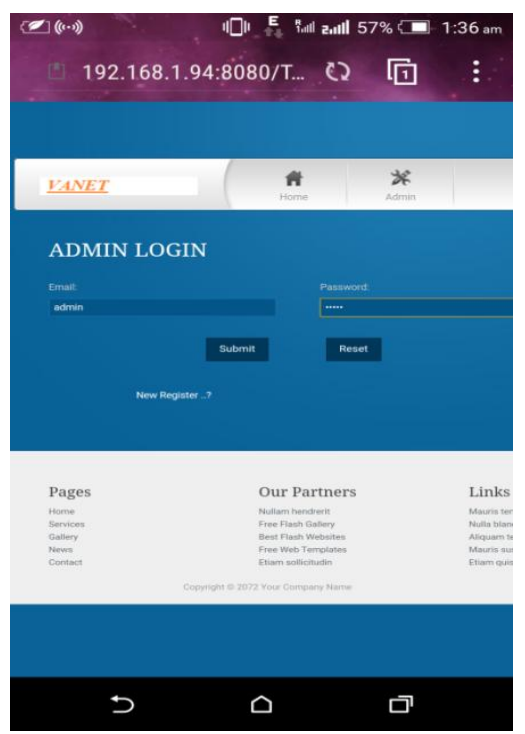

(b) 
Now if any registered user selects user login then he has to provide his email address and password as shown in figure 4(c).

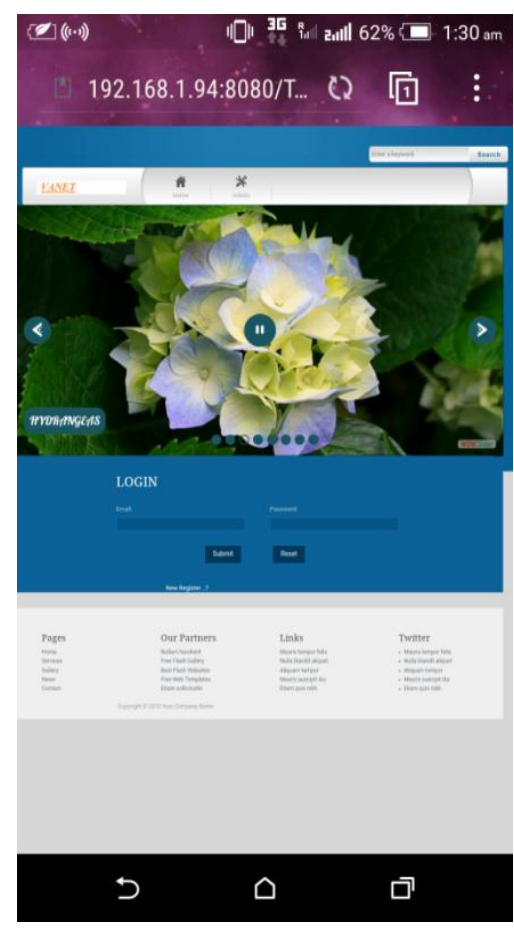

(c)

Application will now ask for start and end address i.e. source and destination of user. For e.g. kkw and nashik road is start and end address respectively as shown in figure 4(d).

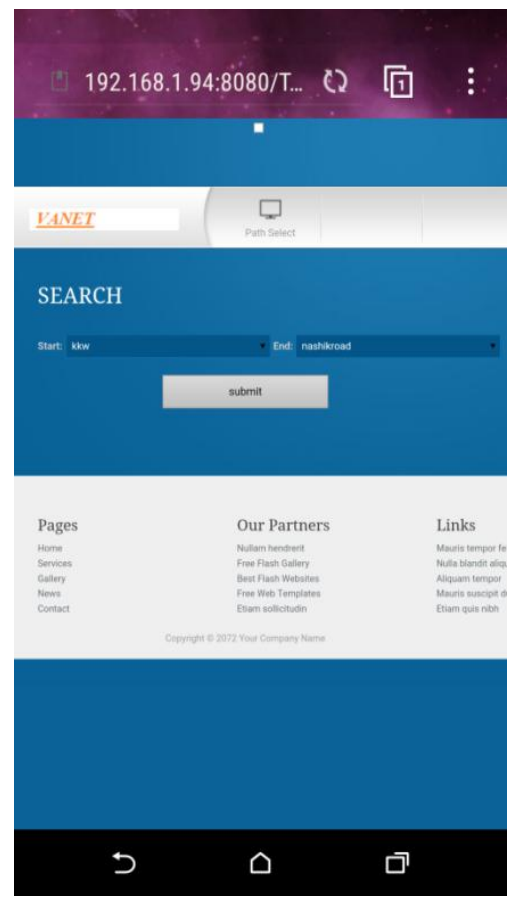

(d)

Then after submitting the request application will provide all the paths from particular source to destination with their respective traffic count as shown in figure 4(e).

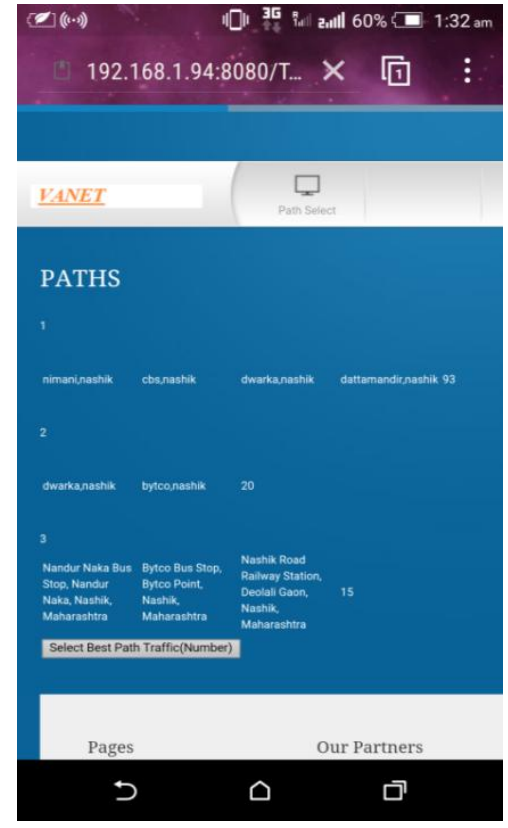

(e)

When the user will ask for best path with minimum traffic, application will provide required path along with google map as shown in figure 4(f).

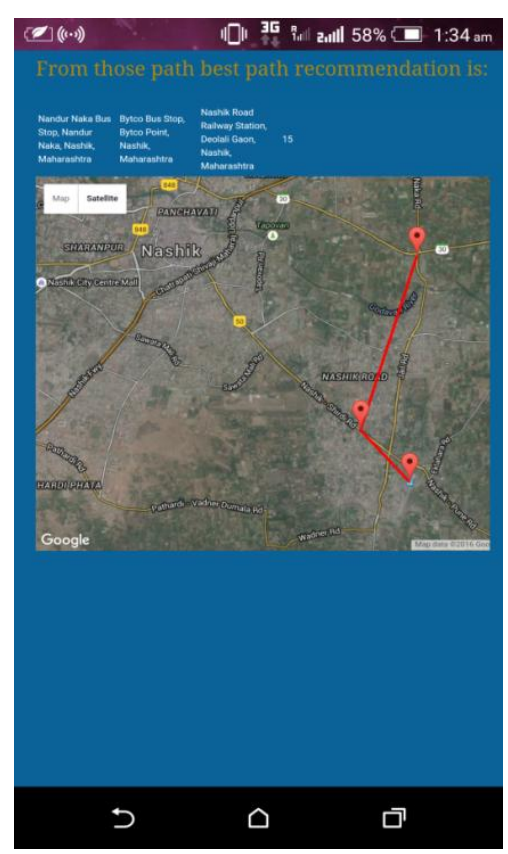

(f)

Fig 4: Screenshot of android application view

\section{CONCLUSION}

This paper gives an overview of past few techniques in the field of vehicular ad hoc networks and presents a real-time intelligent transportation system. Proposed system is based on RFID and ARM controller to minimize traffic congestion with proper signal control. And the android mobile application will help users to find a path with minimum traffic to their destination. This can greatly reduce the problems of road congestion, traffic jams and travel inconvenience. Proposed system can help to avoid congestion and can find better routes 
by real time data. This saves both time and fuel and has significant economic advantages.

\section{FUTURE SCOPE}

System can be made more effective and can be implemented at traffic signal in real life. The RFID reader can be replaced with digital cameras at roadside to increase the efficiency of system. By increasing the range and capability of the components used, the system can become deployable. It can possess more parameters for increasing the applications of system. Changes can be made as per the users requirement and ideas to make it more useful and beneficial for them. All the traffic logs and data can be maintained on cloud which can be helpful to analyze city traffic and beneficial to take various traffic control decisions. Cloud computing and storage solutions provide users and enterprises with various capabilities to store and process their data in third-party data centers.

\section{ACKNOWLEDGEMENT}

The authors acknowledge the breakthrough discussions with the help of K. K. Wagh Institute of Engineering Education and Research, Nasik, India for providing research facilities.

\section{REFERENCES}

[1] Miao Wang, Hangguan Shan, Member, IEEE, Rongxing Lu, Member, IEEE, Ran Zhang, Xuemin (Sherman) Shen, Fellow, IEEE, and Fan Bai, "Real-Time Path Planning Based on Hybrid-VANET-Enhanced Transportation System", IEEE Transactions on vehicular technology, Vol. 64, No. 5, May 2015.

[2] M. Papageorgiou, C. Diakaki, V. Dinopoulou, A. Kotsialos, and Y. Wang, "Review of road traffic control strategies," Proc. IEEE, vol. 91, no. 12, pp. 2043-2067, Dec. 2003.

[3] Texas Transp. Inst. (2007). Texas Transportation Institute: Urban mobility information annual urban mobility report, College Station, TX, USA. [Online]. Available: http://mobility.tamu.edu/ums

[4] H. Hartenstein and K. Laberteaux, VANET: Vehicular Applications and Inter-Networking Technologies. Hoboken, NJ, USA: Wiley, 2010. [Online]. Available: http://www.nhtsa.gov/

[5] T. Hunter, R. Herring, P. Abbeel, and A. Bayen, "Path and travel time inference from GPS probe vehicle data," in Proc. Neural Inf. Process. Syst. Found., Vancouver, BC, Canada, Dec. 2009, pp. 1-8.

[6] A. Khosroshahi, P. Keshavarzi, Z. KoozehKanani, and J. Sobhi, "Acquiring real time traffic information using VANET and dynamic route guidance," in Proc. IEEE Comput., Control Ind. Eng., Wuhan, China, Aug. 2011, pp. 9-13.

[7] R. Lu, X. Lin, and X. Shen, "SPRING: A social-based privacy-preserving packet forwarding protocol for vehicular delay tolerant networks," in Proc. IEEE INFOCOM, San Diego, CA, USA, Mar. 2010, pp. 1-9.
[8] T. Schouwenaars, B. Moor, E. Feron, and J. How, "Mixed integer programming for multi-vehicle path planning," in Proc. Eur. Control Conf., Porto, Portugal, Sep. 2001, pp. 2603-2608

[9] P. Chen, Y. Guo, and W. Chen, "Fuel-saving navigation system in VANETs," in Proc. IEEE Veh. Technol. Conf., Ottawa, ON, Canada, Sep. 2010, pp. 1-5.

[10] Y. Zhao, K. Triantis, D. Teodorovic, and P. Edara, "A travel demand management strategy: The downtown space reservation system," Eur. J. Oper. Res., vol. 205, no. 3, pp. 584-594, Sep. 2010.

[11] M. Kimra, S. Inoue, Y. Taoda, T. Dohi, and Y. Kakuda, "A novel method based on VANET for alleviating traffic congestion in urban transportations," in Proc. IEEE Auton. Decentralized Syst., Mexico City, Mexico, Mar. 2013, pp. 1-7.

[12] M.Wang, H. Liang, R. Zhang, R. Deng, and X. Shen, "Mobility-aware coordinated charging for electric vehicles in VANET-enhanced smart grid," IEEE J. Sel. Areas Commun., vol. 32, no. 7, pp. 1-17, Jul. 2014.

[13] Y. Chung, "Development of an accident duration prediction model on the Korean Freeway Systems," Accid. Anal. Prev., vol. 42, no. 1, pp. 282-289, Jan. 2010.

[14] Gholamreza Vatanian Shanjani* and Somayyeh Jafarali Jassbi, Taxonomy of Intelligent Transportation Systems (VANET): A Survey, Journal of Advances in Computer Research Quarterly ISSN: 2008-6148 Sari Branch, Islamic Azad University, Sari, I.R.Iran, Vol. 5, No. 1, February 2014.

[15] A. Skabardonis and N. Geroliminis, "Real-time monitoring and control on signalized arterials," J. Intell. Transp. Syst.-Technol., Plan., Oper., vol. 12, no. 2, pp. 64-74, May 2008.

[16] Chao Wang, Xi Li, Aili Wang, Feng Yang, Xuehai Zhou, "An Intelligent Transportation System using RFID based Sensors", IET Netw., 2013, Vol. 4, Iss. 3, pp. 185-194.

[17] M. H. Assaf, K. M. Williams, "RFID for Optimisation of Public Transportation System", Globecom 2011 Communications Software, Services and Multimedia Symposium.

[18] Nazmus S. Nafi, Reduan H. Khan, Jamil Y. Khan, Mark Gregory RMIT University Australia, The University of Newcastle Australia, “A Predictive Road Traffic Management System Based on Vehicular Ad-hoc Network", IEEE/CAA Journal Oof Automatica Sinica, Vol. 2, No. 3, July 2014

[19] Huanqi Tao, WencongLiu, Shuangbao Ma, "Intelligent Transportation Systems for Wireless Sensor Networks based on ZigBee", 2010 International Conference on Computer and Communication Technologies in Agriculture Engineering". 